\title{
PReS-FINAL-2236: Continuous autoinflammatory syndromes: a single-center experience in Argentina
}

\author{
RA Russo, MM Katsicas \\ From 20th Pediatric Rheumatology European Society (PReS) Congress \\ Ljubljana, Slovenia. 25-29 September 2013
}

\begin{abstract}
Introduction
Patients with autoinflammatory syndromes may present a clinical course characterized by recurrent, episodic manifestations (such as fever, skin rash or visceral involvement) or they may show a continuous, unremitting disease course with persistent clinical manifestations. Patients with certain diseases, such as CAPS or Blau syndrome, usually present this type of course.
\end{abstract}

\section{Objectives}

To describe the clinical and genetic features of patients with continuous-course autoinflammatory syndromes followed in a tertiary, pediatric hospital.

\section{Methods}

Ad-hoc data bases from our autoinflammatory syndromes clinic were reviewed. Patients attended this clinic between May 2009 and May 2013. Demographic, clinical, laboratory and genetic data were retrieved. Autoinflammatory syndrome was defined as the presence of a chronic, systemic disease with no evidence of malignancy, infection or autoimmunity. Patients with a continuous disease course (persistent clinical manifestations with no free interval and with possible recurrent exacerbations prior to the initiation of therapy) were included in the analysis. Patients with a diagnosis of systemic juvenile arthritis were excluded. Genetic analysis was performed in different locations.

\section{Results}

Fourteen children ( 9 boys) with a continuous disease course were identified among patients with autoinflammatory syndromes. Median age at presentation: 6 months;

\footnotetext{
Immunology \& Rheumatology, Hospital De Pediatría Garrahan, Buenos Aires,
} Argentina
Cite this article as: Russo and Katsicas: PReS-FINAL-2236: Continuous autoinflammatory syndromes: a single-center experience in Argentina. Pediatric Rheumatology 2013 11(Suppl 2):P226. 